\title{
Regional Plan of Integration of South Moravian and Lower Austrian Border Regions
}

\author{
MOJMIR KYSELKA \\ Technical University of Brno \\ Czech Republic
}

\section{INTRODUCTION}

This transborder regional plan represents the final result of the collaboration of three universities: Faculty of Architecture, Technical University of Brno - Czech Republic, Institutes of Regional and Landscape Planning TU Vienna Austria and the Institute of Regional and Environmental Planning, University of Kaiserslautern - Germany. All the participants, students and teachers, architects, urban and regional planners enjoyed the four common workshops both on the Czech and on the Austrian territory, which was divided till 1989 by the "iron curtain". They compared the differences of the local culture in architecture, urban and landscape structure, but found the majority of similar ways of life. This was what created the idea of the transborder zone.

The principal mission of this research work was to widen the knowledge in the area of regional planning and to study and compare the present European status of this discipline, especially in the German speaking countries. The research has shown substantial differences not only between German, Swiss and Austrian methods but also between the methodology of their own provinces or cantons. The study of regional plans of the joint border areas of Germany and France, especially that of PAMINA, which was developed at the Kaiserslautern University and covers the German area of Pfalz-Baden-Wuertenberg and the French area of the Northern Alsace, produced completely new facts. Finally, the very different regional planning methods of the centralized Czech and the decentralized Lower Austrian approach were thoroughly analyzed and applied to the plan of the joint CzechAustrian border regions. All the findings, together with the critical comments were summarized and defended at the end of 1992. Their application continued until the completion of the task in 1994.

Education was the second objective of the research. Ideally, each university should model in their curricula the conditions and situations which will occur in the next five, ten or twenty years to prepare today's students to deal with them, and to apply this knowledge wisely in the future. One of these situations is the gradual integration of European countries and their cooperation specifically in the planning of the border regions. Until recently, the Eastern Europe border regions were separated from the west by a sharp line of "iron curtain". This was the reason for providing research analysis and proposals for the border region between Breclav - Znojmo and Retz - Schrattenberg by the senior students and professors of the Faculty of Architecture and the Faculty of Civil Engineering of TU Bmo and TU Wien and UNI Kaiserslautern. During the three joint seminars held in both countries, it was the work of students which brought dynamic, optimistic, but also realistic aspects into the border region: "AHA" (Autonomia, Hospia, Artia) and "JIZMONOWEI" (JIZni MOrava, Nieder Oesterreich, WEInviertel). Without the thorough involvement of the students it would have been impossible to provide a detailed research and to propose 9 alternatives for the future development.

The third aspect of the project, which evolved during the research was a political one. The creation of a new border with Slovakia and the need for a dialog with Austria were the main factors, why the the scientific board of TU Brno decided to include the project in its programme and fund it. It was also accepted by the TU Wien and by the representatives of the Lower Austrian Regional Government. Towards the end of $1993 \mathrm{Dr}$. Erhard Busek, the Austrian vicechancellor and the Minister of Science and Research personally endorsed the programme. He provided the means for the travelling exhibition, which will in the years 1995-96 inform the citizens of both countries about the plans for the joint rural tourism, based on the existing cultural, natural and recreational wealth of the region while enjoying the local food and wine.

The fourth, totally unexpected contribution of the research was the discovery of the philosophy of the borders. Not only the political borders between the sovereign states, but also the borders between different life styles in general. Both during the investigations and input by schools and universities and also during the ongoing philosophical debates about the substance of our task it was found that the preferred category of separation was not a rigid line, but rather an area and space of transitional shape, characterized 
by continuous engagement and merger. These transitional borders can also be seen between the natural ecosystems, they support the political, cultural and economic prosperity of the Swiss, and are a reminiscence of the former
Liechtenstein domain in the Moravian - Austrian region. The research contributed to enrichment of the knowledge of participants in the field of both rational and emotional values. 